\title{
Estimation of 2-D Noisy Fractional Brownian Motion and its Applications using Wavelets
}

\author{
Jen-Chang Liu ${ }^{\dagger \ddagger}$, Wen-Liang Hwang ${ }^{\ddagger}$, and Ming-Syan Chen ${ }^{\dagger}$ \\ Department of Electrical Engineering, National Taiwan University, Taiwan ${ }^{\dagger}$ \\ Institute of Information Science, Academia Sinica, Taiwan ${ }^{\ddagger}$
}

\begin{abstract}
The 2-D fractional Brownian motion (fBm) model is useful in describing natural scenes and textures. Most fractal estimation algorithms for 2-D isotropic fBm images are simple extensions of the 1-D fBm estimation method. This method does not perform well when the image size is small (say, $32 \times 32$ ). We propose a new algorithm that estimates the fractal parameter from the decay of the variance of the wavelet coefficients across scales. Our method places no restriction on the wavelets. Also, it provides a robust parameter estimation for small noisy fractal images. For image denoising, a Wiener filter is constructed by our algorithm using the estimated parameters and is then applied to the noisy wavelet coefficients at each scale. We show that the averaged power spectrum of the denoised image is isotropic and is a near $\frac{1}{f}$ process. The performance of our algorithm is shown by numerical simulation for both the fractal parameter and the image estimation. Applications on coastline detection and texture segmentation in noisy environment are also demonstrated.
\end{abstract}

EDICS number: IP 1.6

Address : Institute of Information Science, Academia Sinica, Taiwan e-mail: whwang@iis.sinica.edu.tw 


\section{Introduction}

Fractional Brownian motion $(\mathrm{fBm})$ is a non-stationary stochastic model, which has a $1 / f$ spectrum and the statistical self-similar property [15]. For an isotropic 2-D fBm, it has the averaged power spectrum [4][23]

$$
P\left(w_{x}, w_{y}\right)=\frac{\sigma^{2}}{\left(\sqrt{w_{x}^{2}+w_{y}^{2}}\right)^{2 \alpha+2}},
$$

where $\alpha$ is the scaling exponent, $0<\alpha<1$. Other types of $2-\mathrm{D} \mathrm{fBm}$, such as the multi-fractal rough surface [25] and the extended self-similar model [10], also exhibit features controlled by the scaling exponent $\alpha$. Many natural phenomena are found to have $1 / f$ spectrums. Thus, an $\mathrm{fBm}$ provides good mathematical modeling of these phenomena. Moreover, the self-similar property, which means that the statistical measure is invariant to the change of scales, makes $\mathrm{fBm}$ very useful in describing natural scenes and textures. The scaling exponent $\alpha$ has also been shown to be related to the fractal dimension and surface roughness [16]. Many research works have focused on the generation of $\mathrm{fBm}[11][20]$ and estimation of the fractal parameter (scaling exponent) $\alpha[2][8][12][21]$. Among them, the wavelet approach was adopted naturally because the statistical self-similarity properties of an $\mathrm{fBm}$ can be described based on the scaling properties of wavelet transforms. Most of the previous wavelet-based results have depended heavily on the orthogonality and vanishing moment of the wavelet function. They used the approximation that the orthogonal wavelet coefficients are almost white processes. This approximation works only if orthogonal wavelets with high vanishing moment are used. The performance will severely degrade if non-orthogonal wavelets are used. It was shown in [7] that the orthogonality of a wavelet can be discarded if the fractal parameter is estimated from the autocorrelation of the wavelet transform of an $\mathrm{fBm}$. In spite of the comparative performance of the $\mathrm{fBm}$ estimation and denoising methods with the results obtained using orthogonal wavelet transform, the approach in [7] allows fractal estimation and other applications, such as edge detection and in- 
stantaneous frequency analysis, both of which are captured nicely by non-orthogonal wavelet transforms, to be done with one wavelet transform analysis [3][13][14].

In this paper, we will extend the proposed methods in [7] to an isotropic 2-D noisy $\mathrm{fBm}$ image. The extension is not straightforward. Although one can obtain the fractal parameter of an isotropic $\mathrm{fBm}$ by averaging the estimated fractal parameters from several directions using the 1-D fractal parameter estimation algorithm [2][9][12][22], this approach does not work well in practice. Note that when the fBm is embedded in additive white noise environment, it usually requires a sufficient number of sampled points for robust 1-D fractal parameter estimation [7]. Thus, for a small image (say of size less than $64 \times 64$ ), there are not enough pixels in each direction for accurate 1-D fractal parameter estimation. As a result, alternative methods must be developed in order to achieve fractal estimation from a small noisy $\mathrm{fBm}$ image. In this paper, we show that the wavelet transform of an isotropic fBm image at each scale is a twodimensional wide-sense stationary (WSS) process. Thus, fractal parameter estimation can be obtained from two-dimensional wavelet coefficients, even in the case of a small noisy $\mathrm{fBm}$ image. We propose a fractal parameter estimation algorithm which formulates the fractal parameter estimation problem as the characterization of a composite singularity from the autocorrelation of the wavelet transforms of an noisy fBm image. All the related parameters are then solved and estimated using a robust regression method. Our proposed 2-D estimation method is more efficient than those based on averaging the results obtained by applying 1-D estimation method many times on a 2-D fBm image. For fBm image estimation, we apply the Wiener filter to noisy wavelet coefficients at each scale. The "denoised" image is then obtained by means of wavelet reconstruction. Finally, we show that the denoised image is a near $\frac{1}{f}$ process. The proposed parameter estimation and denoising method are applied on problems of coastline detection and texture segmentation.

In Section 2, we derive the properties of the autocorrelation of the wavelet transform of a 2-D noisy fBm. The parameter estimation method is also developed in this section. 
In Section 3, we discuss the image denoising method. In Section 4, simulation results based on these methods are shown. We also demonstrate the applications on coastline detection and texture segmentation. Conclusions are given in the final section.

\section{Fractal Parameter Estimation from the Autocorrelation of 2-D Wavelet Transform}

In this section, we will show that the wavelet transform of an fBm image is a twodimensional WSS process at each scale. Moreover, the variance of the wavelet transformed image at each scale $s$ is proportional to $s^{2 \alpha}$, where $\alpha$ is the fractal parameter of the fBm. Using a similar procedure, we will also prove that the wavelet transform of a white noise image is also stationary in both the horizontal and vertical directions, and that its variance at each scale $s$ is proportional to $s^{-2}$.

The wavelet transform $\mathcal{W}_{s} f_{\alpha}(x, y)$ of a 2-D fBm image $f_{\alpha}(u, v)$ with scaling exponent $\alpha$ is formulated as

$$
\mathcal{W}_{s} f_{\alpha}(x, y)=\iint f_{\alpha}(x-u, y-v) \psi_{s}(u, v) d u d v
$$

where $\psi(u, v)$ is the wavelet, and $\psi_{s}(u, v)=\frac{1}{s^{2}} \psi\left(\frac{u}{s}, \frac{v}{s}\right)$. The autocorrelation of the wavelet transform $\mathcal{W}_{s} f_{\alpha}(x, y)$ at the scale $s$ is derived as follows:

$$
\begin{aligned}
& E\left\{\mathcal{W}_{s} f_{\alpha}(x, y) \mathcal{W}_{s} f_{\alpha}\left(x+\tau_{x}, y+\tau_{y}\right)\right\} \\
= & E\left\{\iint f_{\alpha}(x-u, y-v) \psi_{s}(u, v) d u d v \iint f_{\alpha}\left(x+\tau_{x}-m, y+\tau_{y}-n\right) \psi_{s}(m, n) d m d n\right\} \\
= & \iiint \int E\left\{f_{\alpha}(x-u, y-v) f_{\alpha}\left(x+\tau_{x}-m, y+\tau_{y}-n\right)\right\} \psi_{s}(u, v) \psi_{s}(m, n) d u d v d m d n,
\end{aligned}
$$

where $\tau_{x}$ and $\tau_{y}$ are shifts in the horizontal and vertical directions, respectively. Note that the autocorrelation of the fBm image is [11]

$$
\begin{aligned}
& E\left\{f_{\alpha}(x-u, y-v) f_{\alpha}\left(x+\tau_{x}-m, y+\tau_{y}-n\right)\right\} \\
= & \sigma_{\alpha}{ }^{2}\left\{\left[(x-u)^{2}+(y-v)^{2}\right]^{\alpha}+\left[\left(x+\tau_{x}-m\right)^{2}+\left(y+\tau_{y}-n\right)^{2}\right]^{\alpha}\right. \\
& \left.-\left[\left(\tau_{x}-m+u\right)^{2}+\left(\tau_{y}-n+v\right)^{2}\right]^{\alpha}\right\},
\end{aligned}
$$


where $\sigma_{\alpha}^{2}$ is a constant. Furthermore, from the properties of wavelets [14], the following equation must be satisfied:

$$
\iint \psi_{s}(u, v) d u d v=0
$$

Replacing (3),(4) into (2), we can simplify the above to

$$
\iiint \int-\sigma_{\alpha}{ }^{2}\left|\left(\tau_{x}-m+u, \tau_{y}-n+v\right)\right|^{2 \alpha} \psi_{s}(u, v) \psi_{s}(m, n) d u d v d m d n
$$

where $|(u, v)|=\sqrt{\left(u^{2}+v^{2}\right)}$. By changing of variables with $p=m-u$ and $q=n-v$, the above equation can be further simplified to:

$$
\begin{aligned}
& \iiint \int-\sigma_{\alpha}{ }^{2}\left|\left(\tau_{x}-p, \tau_{y}-q\right)\right|^{2 \alpha} \psi_{s}(u, v) \psi_{s}(p+u, q+v) d u d v d p d q \\
= & \iint-\sigma_{\alpha}{ }^{2}\left|\left(\tau_{x}-p, \tau_{y}-q\right)\right|^{2 \alpha} \frac{1}{s^{2}} \Lambda\left(\frac{p}{s}, \frac{q}{s}\right) d p d q \\
= & R_{\mathcal{W}_{s} f_{\alpha}}\left(\tau_{x}, \tau_{y}\right)
\end{aligned}
$$

where $\Lambda(x, y)=\iint \psi(u, v) \psi(u+x, v+y) d u d v$. From the above equation, we know that the autocorrelation of the wavelet transform of a 2-D fBm at each scale depends only on the shift parameters $\tau_{x}$ and $\tau_{y}$. Therefore, the wavelet transform of a $2-\mathrm{D} \mathrm{fBm}$ is a WSS process at each scale [24]. Replacing $\tau_{x}=0$ and $\tau_{y}=0$ in (5), we have

$$
R_{\mathcal{W}_{s} f_{\alpha}}(0,0)=\iint-\sigma_{\alpha}{ }^{2}|(p, q)|^{2 \alpha} \frac{1}{s^{2}} \Lambda\left(\frac{p}{s}, \frac{q}{s}\right) d p d q \text {. }
$$

Let $u=p / s$ and $v=q / s$; the above equation becomes

$$
R_{\mathcal{W}_{s} f_{\alpha}}(0,0)=-\sigma_{\alpha}{ }^{2} s^{2 \alpha} \iint|(u, v)|^{2 \alpha} \Lambda(u, v) d u d v=s^{2 \alpha} K_{p}
$$

where $K_{p}$ depends on $\alpha$ and the wavelet, and $K_{p}$ is a fixed constant given the wavelet transform of a 2-D fBm image. The variance of wavelet transform at each scale $s$ changes according to $s^{2 \alpha}$. This variance progression provides a method to estimate the scaling exponent $\alpha$, and this method works for orthogonal or non-orthogonal wavelets because in our deduction, we only require that the wavelets satisfy (4). 
Following a similar procedure, the formula of the autocorrelation of the wavelet transform of the 2-D white noise $n(u, v)$ is derived as

$$
\begin{aligned}
& E\left\{\mathcal{W}_{s} n(x, y) \mathcal{W}_{s} n\left(x+\tau_{x}, y+\tau_{y}\right)\right\} \\
= & \iint \sigma_{n}{ }^{2} \delta\left(\tau_{x}-p, \tau_{y}-q\right) \frac{1}{s^{2}} \Lambda\left(\frac{p}{s}, \frac{q}{s}\right) d p d q \\
= & R_{\mathcal{W}_{s} n}\left(\tau_{x}, \tau_{y}\right),
\end{aligned}
$$

where $\sigma_{n}{ }^{2}$ is the noise variance. Again, by replacing $\tau_{x}=0$ and $\tau_{y}=0$, we obtain

$$
R_{\mathcal{W}_{s} n}(0,0)=\sigma_{n}{ }^{2} \frac{1}{s^{2}} \Lambda(0,0)=\frac{1}{s^{2}} K_{n}
$$

where $K_{n}$ is determined by the noise variance and wavelet. The variance of wavelet transform at scale $s$ of the white noise changes proportionally to $s^{-2}$.

Assume that $z(u, v)=f_{\alpha}(u, v)+n(u, v)$ is a 2-D $\mathrm{fBm}$ embedded in white noise. Because the wavelet transform is a linear operation, we can combine the result of wavelet transform for 2-D fBm and white noise by means of addition. The autocorrelation of the wavelet transform of the noisy fBm is the summation of (5) and (7):

$$
\begin{aligned}
& R_{\mathcal{W}_{s} z}\left(\tau_{x}, \tau_{y}\right) \\
= & \iint\left[-\sigma_{\alpha}{ }^{2}\left|\left(\tau_{x}-p, \tau_{y}-q\right)\right|^{2 \alpha}+\sigma_{n}{ }^{2} \delta\left(\tau_{x}-p, \tau_{y}-q\right)\right] \frac{1}{s^{2}} \Lambda\left(\frac{p}{s}, \frac{q}{s}\right) d p d q \\
= & {\left[-\sigma_{\alpha}{ }^{2}|(u, v)|^{2 \alpha}+\sigma_{n}{ }^{2} \delta(u, v)\right] * \Lambda_{s}\left(\tau_{x}, \tau_{y}\right), }
\end{aligned}
$$

where $\Lambda_{s}\left(\tau_{x}, \tau_{y}\right)=\frac{1}{s^{2}} \Lambda\left(\frac{\tau_{x}}{s}, \frac{\tau_{y}}{s}\right)$. In fact, (9) is the wavelet transform of $-\sigma_{\alpha}{ }^{2}|(u, v)|^{2 \alpha}+$ $\sigma_{n}{ }^{2} \delta(u, v)$ with wavelet $\Lambda(u, v)$, which has a vanishing moment two times greater than $\psi(u, v)$. It is worth noting that $-\sigma_{\alpha}{ }^{2}|(u, v)|^{2 \alpha}+{\sigma_{n}}^{2} \delta(u, v)$ has a composite singularity at $(0,0)$, which is the superposition of an isotropic peak and a Dirac. The problem of parameter estimation can then be related to the detection and characterization of singularities [13]. Taking $\left(\tau_{x}, \tau_{y}\right)=(0,0)$, the variance of the wavelet transform of $z(u, v)$ is

$$
R_{\mathcal{W}_{s} z}(0,0)=K_{p} s^{2 \alpha}+K_{n} s^{-2}
$$


for $0<\alpha<1$ and $K_{n}, K_{p} \geq 0$. The above variance progression formula does not depend on wavelets that have more vanishing moments, which only influences the decay of the autocorrelation $R_{\mathcal{W}_{s} z}\left(\tau_{x}, \tau_{y}\right)$ at $\left(\tau_{x}, \tau_{y}\right) \neq(0,0)$.

In practice, it is sufficient to estimate the parameters $K_{p}, K_{n}$ and $\alpha$ from the dyadic scales. $K_{p}, K_{n}$, and $\alpha$ in Equation (10) can be obtained from any three dyadic scales. However, to get a robust numerical result, we shall estimate these parameters from as many different scales as possible. For dyadic scales $s=2^{j}, j=1, \ldots, m$, we find the parameters $K_{n}, K_{p}$, and $\beta=2^{2 \alpha}$ that are the solution of the following constrained nonlinear minimization problem:

$$
\begin{gathered}
f\left(K_{p}, K_{n}, \beta\right)=\min \sum_{j=1}^{m}\left(K_{p} \beta^{j}+K_{n} 2^{-2 j}-R_{\mathcal{W}_{2 j} z}(0,0)\right)^{2} \\
\text { subject to } \\
\qquad \leq K_{n} \leq \frac{\sum_{j=1}^{m} R_{\mathcal{W}_{2 j} z}(0)}{\sum_{j=1}^{m} 2^{-2 j}} \\
0 \leq K_{p} \leq \frac{\sum_{j=1}^{m} R_{\mathcal{W}_{2 j} z}(0)}{\sum_{j=1}^{m} 1} \\
1 \leq \beta \leq 4 \\
n \geq 3 .
\end{gathered}
$$

In the nonlinear minimization problem as in (11), we need to solve three parameters $K_{n}, K_{p}$, and $\beta$ to fit the variance of wavelet transform at each scale. But from our observations in experiments and from those given in another report [8], we know that the variances at some scales are not stable. This may introduce significant bias in the final estimation result. The authors in [8] tried to exclude the first scale, or the first two scales, and claimed to have better results. The method proposed is not a systematic 
method generally. Therefore we change our least mean square formula in (11) into a least median of squares regression one :

$$
f\left(K_{p}, K_{n}, \beta\right)=\min \operatorname{med}_{j}\left(K_{p} \beta^{j}+K_{n} 2^{-2 j}-R_{\mathcal{W}_{2 j} z}(0,0)\right)^{2}
$$

The least median of squares algorithm has been claimed to resist the effect of nearly $50 \%$ of contamination in data [18]. However, it has the drawback of low computation

efficiency. In practical computation, we first calculate the solution of $K_{n}, K_{p}$, and $\beta$ from variances from any three scales. All possible combinations of any three scales are included. Then, the median of the square terms in (12) is found for all combinations. We choose the combination with the minimal median. We next include half of the scales whose square terms are less than those of the other half. Finally, a constrained nonlinear minimization algorithm is applied to the data of these scales to find the solution of $K_{n}, K_{p}$ and $\beta$. The nonlinear minimization formula becomes

$$
f\left(K_{p}, K_{n}, \beta\right)=\min \sum_{j \in J}\left(K_{p} \beta^{j}+K_{n} 2^{-2 j}-R_{\mathcal{W}_{2 j} z}(0,0)\right)^{2},
$$

where $J$ is the set that contains the selected scales from the least median of squares method.

\subsection{Optimization by the Penalty Method}

There are many algorithms for solving of a constrained nonlinear minimization problem. We have used the internal penalty method in our experiments. The internal penalty method transforms the constrained problem into an unconstrained problem so that the minimization can be solved easily [1].

Let $N=\frac{\sum_{j=1}^{m} R_{\mathcal{W}_{2 j}}(0,0)}{\sum_{j=1}^{m} 2^{-2 j}}$ and $P=\frac{\sum_{j=1}^{m} R_{\mathcal{W}_{2 j} z}(0,0)}{\sum_{j=1}^{m} 1}$. The penalty function of equation (13) is

$$
\phi_{r}\left(K_{p}, K_{n}, \beta\right)=f\left(K_{p}, K_{n}, \beta\right)+r\left(\frac{1}{N-K_{n}}+\frac{1}{K_{n}}+\frac{1}{P-K_{p}}+\frac{1}{K_{p}}+\frac{1}{4-\beta}+\frac{1}{\beta-1}\right),
$$


where $f\left(K_{p}, K_{n}, \beta\right)=\sum_{j \in J}\left(K_{p} \beta^{j}+K_{n} 2^{-2 j}-R_{\mathcal{W}_{2 j} z}(0,0)\right)^{2}$ is the objective function, $r>0$ is the penalty parameter, and the terms following $r$ are obtained from the constraints (11). We can find an initial $K_{n}, K_{p}$, and $\beta$ from any three scales, and calculate an initial $r$ as the ratio of the objective function $f\left(K_{p}, K_{n}, \beta\right)$ to the penalty terms. A local minimization technique, such as the conjugate gradient method, can be used to find the local minimum of $\phi_{r}\left(K_{p}, K_{n}, \beta\right)$, which occurs at $K_{p}^{*}, K_{n}^{*}$, and $\beta^{*}$. Then, $r$ can be multiplied by a constant less than 1 . These new parameters are used to find the local minimum of $\phi_{r}$ again. This process can be iterated until the desired accuracy is reached.

\section{Fractal Image Estimation}

Although several algorithms have been proposed to estimate the parameters of a noisy fBm image [9], few works have focused on the reconstruction of an fBm image from a noisy environment. Extension of 1-D fBm algorithms of signal reconstruction to 2-D fBm image denoising might be straightforward. However, little work has been reported in the literature. In the classic algorithm of fBm signal reconstruction given in [21], the authors made an assumption that the wavelet transform of an $\mathrm{fBm}$ is white noise. The assumption is an approximation that depends on the number of vanishing moments of orthogonal wavelets. Extension of their algorithm to the 2-D case can be done easily and is thus omitted here. In this section, we will propose an fBm image estimation algorithm that places no constraints on the orthogonality of wavelets.

Since we have shown that the wavelet transform of a 2-D noisy fBm is a WSS process at each scale, Wiener filtering can be applied to each scale. Note that in Section 2, the autocorrelation of the wavelet transform $\mathcal{W}_{s} f_{\alpha}(x, y)$ of a $2-\mathrm{D}$ fBm at scale $s$ was

$$
R_{\mathcal{W}_{s} f_{\alpha}}\left(\tau_{x}, \tau_{y}\right)=\iint-\sigma_{\alpha}{ }^{2}\left|\left(\tau_{x}-p, \tau_{y}-q\right)\right|^{2 \alpha} \frac{1}{s^{2}} \Lambda\left(\frac{p}{s}, \frac{q}{s}\right) d p d q
$$

By simple calculation, the power spectra $S_{s} f_{\alpha}\left(w_{x}, w_{y}\right)$ of $\mathcal{W}_{s} f_{\alpha}(x, y)$ is the Fourier 
transform of (14), and we obtain

$$
S_{s} f_{\alpha}\left(w_{x}, w_{y}\right)=\frac{\sigma_{\alpha}^{2} 2 \sqrt{\pi} \Gamma(2 \alpha+2) \sin (\pi \alpha)}{{\sqrt{w_{x}^{2}+w_{y}^{2}}}^{2 \alpha+2}} \hat{\Lambda}\left(s w_{x}, s w_{y}\right),
$$

where $\hat{\Lambda}\left(w_{x}, w_{y}\right)$ is the Fourier transform of $\Lambda\left(w_{x}, w_{y}\right)$. Recall that the autocorrelation of the wavelet transform of $2-\mathrm{D}$ white noise is

$$
R_{\mathcal{W}_{s} n}\left(\tau_{x}, \tau_{y}\right)=\iint{\sigma_{n}}^{2} \delta\left(\tau_{x}-p, \tau_{y}-q\right) \frac{1}{s^{2}} \Lambda\left(\frac{p}{s}, \frac{q}{s}\right) d p d q,
$$

and that its Fourier transform is

$$
S_{s} n\left(w_{x}, w_{y}\right)=\sigma_{n}{ }^{2} \hat{\Lambda}\left(s w_{x}, s w_{y}\right)
$$

Note that $\mathcal{W}_{s} f_{\alpha}(x, y)$ and $\mathcal{W}_{s} n(x, y)$ are uncorrelated, since $f_{\alpha}(x, y)$ and $n(x, y)$ are uncorrelated; the frequency response of the Wiener filter for the wavelet transform of a noisy $\mathrm{fBm}$ is an isotropic function of the frequency and has the following form :

$$
\begin{gathered}
H_{s}\left(w_{x}, w_{y}\right)=\frac{S_{s} f_{\alpha}\left(w_{x}, w_{y}\right)}{S_{s} f_{\alpha}\left(w_{x}, w_{y}\right)+S_{s} n\left(w_{x}, w_{y}\right)} \\
=\frac{\frac{\sigma_{\alpha}{ }^{2} 2 \sqrt{\pi} \Gamma(2 \alpha+2) \sin (\pi \alpha)}{{\sqrt{w_{x}^{2}+w_{y}^{2}}}^{2 \alpha+2}} \hat{\Lambda}\left(s w_{x}, s w_{y}\right)}{\left(\frac{\sigma_{\alpha}{ }^{2} 2 \sqrt{\pi} \Gamma(2 \alpha+2) \sin (\pi \alpha)}{{\sqrt{w_{x}^{2}+w_{y}^{2}}}^{2 \alpha+2}}+{\sigma_{n}}^{2}\right) \hat{\Lambda}\left(s w_{x}, s w_{y}\right)} \\
=\frac{\sigma_{\alpha}^{2} 2 \sqrt{\pi} \Gamma(2 \alpha+2) \sin (\pi \alpha)}{\sigma_{\alpha}{ }^{2} 2 \sqrt{\pi} \Gamma(2 \alpha+2) \sin (\pi \alpha)+\sigma_{n}^{2}{\sqrt{w_{x}^{2}+w_{y}^{2}}}^{2 \alpha+2}} .
\end{gathered}
$$

As shown by the above calculation, the Wiener filter appears to be scale indepedent. Our denoising algorithm first applies the proposed fractal parameter estimation method for parameters $\alpha$ and $\sigma_{\alpha}^{2}$ in equation (18), then the wavelet coefficients of the noisy fBm at each scale are passed through the corresponding Wiener filter. After all, the wavelet reconstruction produced a denoised fBm image.

Now, we will show that the power spectrum of the denoised fBm image is isotropic and is a near $\frac{1}{f}$ process. Let us take Mallat and Zhong's approach [14]. Let the horizontal wavelet $\psi^{1}(x, y)$ and vertical wavelet $\psi^{2}(x, y)$ be given by

$$
\psi^{1}(x, y)=\psi(x) 2 \phi(2 y), \quad \psi^{2}(x, y)=2 \phi(2 x) \psi(y)
$$


respectively, where $\psi(x)$ is a wavelet which is the derivative of a smoothing function. At each scale $s$, a coarse image and two detail images, which represent the horizontal and vertical details, are generated.

In our denoising algorithm, the Wiener filter is applied to the wavelet coefficients of the noisy $\mathrm{fBm}$ at each scale, and then the denoised image $f^{e}(u, v)$ is recovered by means of wavelet reconstruction :

$$
f^{e}(u, v)=\sum_{s}\left(h_{s}{ }^{1} * \mathcal{W}_{s}{ }^{1} x * \chi_{s}{ }^{1}(u, v)+{h_{s}}^{2} * \mathcal{W}_{s}{ }^{2} x * \chi_{s}{ }^{2}(u, v)\right)
$$

where $\chi^{1}(u, v)$ and $\chi^{2}(u, v)$ are the reconstruction wavelets, $\chi_{s}(u, v)=\frac{1}{s^{2}} \chi\left(\frac{u}{s}, \frac{v}{s}\right)$, and $h_{s}{ }^{1}$ and $h_{s}{ }^{2}$ are the impulse response of the Wiener filter for the horizontal and vertical wavelet coefficients. It is easy to see from (18) that $h_{s}{ }^{1}=h_{s}{ }^{2}$. Without loss of generality, we will use the dyadic wavelet transform. Since $f^{e}(u, v)$ is the output of a sequence of linear operation, its power spectrum can be written as

$$
\begin{aligned}
S_{f e}\left(w_{x}, w_{y}\right)= & S_{x}\left(w_{x}, w_{y}\right)\left|H_{s}\left(w_{x}, w_{y}\right)\right|^{2} \sum_{j \in Z}\left|\hat{\psi}^{1}\left(2^{j} w_{x}, 2^{j} w_{y}\right) \hat{\chi^{1}}\left(2^{j} w_{x}, 2^{j} w_{y}\right)\right|^{2} \\
& +\left|\hat{\psi^{2}}\left(2^{j} w_{x}, 2^{j} w_{y}\right) \hat{\chi}^{2}\left(2^{j} w_{x}, 2^{j} w_{y}\right)\right|^{2}
\end{aligned}
$$

where $S_{x}\left(w_{x}, w_{y}\right)$ is the average power spectrum of the noisy $\mathrm{fBm}$.

To show that the denoised image is a near $\frac{1}{f}$ process, we first deal with the term $\sum_{j \in Z}\left(\left|\hat{\psi}^{1}\left(2^{j} w_{x}, 2^{j} w_{y}\right) \hat{\chi}^{1}\left(2^{j} w_{x}, 2^{j} w_{y}\right)\right|^{2}+\left|\hat{\psi}^{2}\left(2^{j} w_{x}, 2^{j} w_{y}\right) \hat{\chi}^{2}\left(2^{j} w_{x}, 2^{j} w_{y}\right)\right|^{2}\right)$. Some re- 
lated results can be found in [14], and we list them below for convenience :

$$
\begin{aligned}
& |\hat{\phi}(w)| \leq 1 \\
& |H(w)|^{2} \leq 1 \\
& |\hat{\phi}(2 w)|=|H(w)||\hat{\phi}(w)| \\
& \sum_{j \in Z}\left(\hat{\psi}^{1}\left(2^{j} w_{x}, 2^{j} w_{y}\right) \hat{\chi}^{1}\left(2^{j} w_{x}, 2^{j} w_{y}\right)+\hat{\psi}^{2}\left(2^{j} w_{x}, 2^{j} w_{y}\right) \hat{\chi}^{2}\left(2^{j} w_{x}, 2^{j} w_{y}\right)\right)=1, \\
& G(w) K(w)+|H(w)|^{2}=1 \\
& L(w)=\frac{1+|H(w)|^{2}}{2}, \\
& \left|\hat{\psi}^{1}\left(2 w_{x}, 2 w_{y}\right) \hat{\chi}^{1}\left(2 w_{x}, 2 w_{y}\right)\right|^{2}=\left|G\left(w_{x}\right) K\left(w_{x}\right) L\left(w_{y}\right)\right|^{2}\left|\hat{\phi}\left(w_{x}\right)\right|^{4}\left|\hat{\phi}\left(w_{y}\right)\right|^{4}, \\
& \left|\hat{\psi}^{2}\left(2 w_{x}, 2 w_{y}\right) \hat{\chi}^{2}\left(2 w_{x}, 2 w_{y}\right)\right|^{2}=\left|G\left(w_{y}\right) K\left(w_{y}\right) L\left(w_{x}\right)\right|^{2}\left|\hat{\phi}\left(w_{x}\right)\right|^{4}\left|\hat{\phi}\left(w_{y}\right)\right|^{4} .
\end{aligned}
$$

Using (24), the lower bound is

$$
\begin{aligned}
& \sum_{j \in Z}\left(\left|\hat{\psi}^{1}\left(2^{j} w_{x}, 2^{j} w_{y}\right) \hat{\chi}^{1}\left(2^{j} w_{x}, 2^{j} w_{y}\right)\right|^{2}+\left|\hat{\psi^{2}}\left(2^{j} w_{x}, 2^{j} w_{y}\right) \hat{\chi}^{2}\left(2^{j} w_{x}, 2^{j} w_{y}\right)\right|^{2}\right) \\
& \quad \geq\left|\sum_{j \in Z}\left(\hat{\psi}^{1}\left(2^{j} w_{x}, 2^{j} w_{y}\right) \hat{\chi}^{1}\left(2^{j} w_{x}, 2^{j} w_{y}\right)+\hat{\psi}^{2}\left(2^{j} w_{x}, 2^{j} w_{y}\right) \hat{\chi}^{2}\left(2^{j} w_{x}, 2^{j} w_{y}\right)\right)\right|^{2}=1
\end{aligned}
$$


The upper bound is derived from the above relations step by step :

$$
\begin{aligned}
\sum_{j \in Z}\left(\left|\hat{\psi}^{1}\left(2^{j} w_{x}, 2^{j} w_{y}\right) \hat{\chi}^{1}\left(2^{j} w_{x}, 2^{j} w_{y}\right)\right|^{2}+\left|\hat{\psi^{2}}\left(2^{j} w_{x}, 2^{j} w_{y}\right) \hat{\chi^{2}}\left(2^{j} w_{x}, 2^{j} w_{y}\right)\right|^{2}\right) \\
=\sum_{j \in Z}\left(\left|\hat{\phi}\left(2^{j-1} w_{x}\right)\right|^{4}\left|\hat{\phi}\left(2^{j-1} w_{y}\right)\right|^{4}\left[\left|G\left(2^{j-1} w_{x}\right) K\left(2^{j-1} w_{x}\right) L\left(2^{j-1} w_{y}\right)\right|^{2}\right)\right. \\
\left.\quad+\left|G\left(2^{j-1} w_{y}\right) K\left(2^{j-1} w_{y}\right) L\left(2^{j-1} w_{x}\right)\right|^{2}\right] \\
=\sum_{j \in Z}\left(| \hat { \phi } ( 2 ^ { j - 1 } w _ { x } ) | ^ { 4 } | \hat { \phi } ( 2 ^ { j - 1 } w _ { y } ) | ^ { 4 } \left[\left[1-\left|H\left(2^{j-1} w_{x}\right)\right|^{2}\right]^{2}\left[\frac{1+\left|H\left(2^{j-1} w_{y}\right)\right|^{2}}{2}\right]^{2}\right.\right. \\
\left.\left.\quad+\left[1-\left|H\left(2^{j-1} w_{y}\right)\right|^{2}\right]^{2}\left[\frac{1+\left|H\left(2^{j-1} w_{x}\right)\right|^{2}}{2}\right]^{2}\right]\right) \\
\leq \sum_{j \in Z}\left(\left|\hat{\phi}\left(2^{j-1} w_{x}\right)\right|^{2}\left[1-\left|H\left(2^{j-1} w_{x}\right)\right|^{2}\right]+\left|\hat{\phi}\left(2^{j-1} w_{y}\right)\right|^{2}\left[1-\left|H\left(2^{j-1} w_{y}\right)\right|^{2}\right]\right) \\
=\sum_{j \in Z}\left(\left(\left|\hat{\phi}\left(2^{j-1} w_{x}\right)\right|^{2}-\left|\hat{\phi}\left(2^{j} w_{x}\right)\right|^{2}\right)+\left(\left|\hat{\phi}\left(2^{j-1} w_{y}\right)\right|^{2}-\left|\hat{\phi}\left(2^{j} w_{y}\right)\right|^{2}\right)\right) \\
\leq \lim _{w_{x} \rightarrow 0}\left|\hat{\phi}\left(w_{x}\right)\right|^{2}-\lim _{w_{x} \rightarrow \infty}\left|\hat{\phi}\left(w_{x}\right)\right|^{2}+\lim _{w_{y} \rightarrow 0}\left|\hat{\phi}\left(w_{y}\right)\right|^{2}-\lim _{w_{y} \rightarrow \infty}\left|\hat{\phi}\left(w_{y}\right)\right|^{2}=2 .
\end{aligned}
$$

We can see that the summation term is between the upper and lower bounds; therefore, we have recoverd a near $\frac{1}{f}$ process.

Finally, we make a comparison between our algorithm and the spatial-domain estimation. Wiener filtering is equivalent to the spatial-domain minimum MSE estimation. Due to the non-stationarity of $\mathrm{fBm}$, direct application of Wiener filtering to the noisy $\mathrm{fBm}$ is extermely computationally complex in the spatial domain, since it involves the factorization of a correlation matrix that is not Toeplitz. However, our approach is to apply Wiener filter at each scale in the wavelet domain, in which the noisy $\mathrm{fBm}$ is stationary as proved. Although the cross-scale depedencies are ignored in our method, our approach is more computationally efficient. 


\section{Simulation Results and Applications}

In this section, we will first demonstrate the simulation results of our algorithms. Then, the applications on coastline detection and texture segmentation are shown.

\subsection{Simulation results}

For the simulation process, the discrete version of the isotropic 2-D fBm synthesis was given by [11]. The increments of the 2-D fBm are first synthesized by discrete Fourier transform, and then the fBm image is added from the incremental values. This method cannot produce 2-D fBm images with exact fBm statistics, but is claimed by the authors to have almost perfect $\mathrm{fBm}$ statistics and fast implementation. A constant parameter $\sigma_{\alpha}^{2}$ is set as 0.5 in the synthesis process. $64 \mathrm{fBm}$ realizations of image size $256 \times 256$, with each scaling exponent $\alpha=0.2,0.5$, and 0.8 are generated. Smaller image sizes of $128 \times 128,64 \times 64$, and $32 \times 32$ are generated by cutting out the central part of the $256 \times 256$ images. Note that the above generated discrete $2-\mathrm{D} \mathrm{fBm}$ is the periodic sampling of the continuous $\mathrm{fBm}$, that is, $f_{\alpha}[m, n]=f_{\alpha}\left(m T_{s}, n T_{s}\right)$, where $T_{s}$ is the sampling period. When we take the discrete sampling $\mathrm{fBm}$ as the input to the discrete wavelet transform [13], our derivation of equation (9) based on the continuous wavelet transform would be slightly biased on the fine scales, according to the extension of the results shown in [5]. This bias effect would be reduced in our parameter estimation process by the use of least median of squares method.

In our implementation of wavelet transform, we followed the approach described in [13][19], where no decimation was applied to the detailed images in both the horizontal and vertical directions. We then estimated the scaling exponent $\alpha$ in both directions from the detailed images. They were expected to be close in magnitude because we used

the isotropic 2-D fBm images, which had the same scaling exponent in all directions statistically. We then took the average of the scaling exponents in these two directions as the scaling exponent of the whole fBm image. In all the experiments, we adopted two 
wavelets, the Haar wavelet and Mallat wavelet, for comparison of filter performance. An image size of $N \times N$ was decomposed up to $\log _{2} N$ scales. Using the least median of squares method, only the data on half of the scales were selected. $K_{n}, K_{p}$ and $\alpha$ were calculated from the data of the selected scales using internal penalty method. As a comparison, we implemented the Wornell and Oppenheim's 1-D fBm estimation algorithm [21] by using Haar wavelet to estimate the fractal parameters of the 2-D fBm images. For an $N \times N$ image, we estimated the fractal parameters of $N$ 1-D traces both along the horizontal and vertical directions. The total $2 N$ estimated values are then averaged to obtain an estimation of the image.

White noise was added to the fBm images so that the SNR(Signal-to-Noise Ratio) was $5 d B$. The mean and root mean square (RMS) errors of the estimated $\hat{\alpha}$ are plotted in Figs. 1 to 2 as a function of the image size for various values of $\alpha$. From the results of parameter estimation of clean fBm images shown in Fig. 1, we can estimate the scaling exponent $\alpha$ precisely for image sizes larger than $128 \times 128$. The degree of the RMS error is about $10^{-2}$. This result is comparable to that of another proposed method [9], in which the same 2-D fBm generation process was used. As reported in [9], the underestimation of $\alpha$ with a true value 0.8 was also observed by our experiments. The performance of the Haar wavelet was slightly better than that of the Mallat wavelet because Mallat wavelet has longer support, which introduces unwanted boundary effects in smaller images. In the case of a noisy environment, our method still estimates $\alpha$ well for image sizes larger than $128 \times 128$. The estimation error is about $10^{-1}$ worse than that in the case of clean image, showing the robustness of our method to added noise. In all cases, our method always produces estimates of $\alpha$ that are distinguishable from each other if their true values are originally different. This is a good property if we do not require precise estimation, but robust estimation that can still distinguish one fBm region from another, for example, in the application of texture image segmentation [22]. The results show that our proposed 2-D estimation method outperforms 1-D estimation method for small images. The computational complexity of 2-D estimation is much less 
than that of the 1-D estimation, since the 1-D estimation has to be applied to several 1-D traces in the image.

The performance of the image denoising algorithm described in Section 3 was also evaluated. In order to distinguish the error introduced by parameter estimation and the image denoising algorithm, we set a prior the true parameters $\sigma_{\alpha}{ }^{2}$ and ${\sigma_{n}}^{2}$ in the Wiener filter formula (18) in the experiments. The Wiener filter was applied to each scale of wavelet transform. Then, the denoised fBm image was generated by means of wavelet synthesis of the filtered wavelet transform images. Sixty-four realizations of $\mathrm{fBm}$ images, with sizes of $256 \times 256$ and $128 \times 128$, and scaling exponents $\alpha$ of 0.8 , 0.5 , and 0.2, were used. The SNR gain, which is the reconstructed image's SNR minus the original SNR, was measured by taking the average of 64 SNR gains for each case described above. The Mallat wavelet [14] was used in our experiments. The results are shown in Fig. 3. Images of size $256 \times 256$ have about 2 to $3 \mathrm{~dB}$ more SNR gains than those of size $128 \times 128$ in the case of $\alpha=0.8$ and 0.5 . The SNR gain of $\alpha=0.8$ is higher than that of $\alpha=0.5$ for about $5 \mathrm{~dB}$, and $\alpha=0.5$ is higher than $\alpha=0.2$ for about 5 to $6 \mathrm{~dB}$. The degrading of the denoising effect for small $\alpha$ values is due to the smoothing effect of the Wiener filter. The fBm images with lower $\alpha$ values represent rougher surfaces [16], and exhibit similar behavior with respect to noises. Therefore, the Wiener filter not only smoothes out the added noises, but also smoothes out the original roughness of the $\mathrm{fBm}$ images. The low SNR images have better SNR gains after denoising.

For visual evaluation, we present some sample figures of image denoising in Figs. 4 to 6 . The $256 \times 256 \mathrm{fBm}$ images with $\alpha=0.8,0.5$, and 0.2 , were added with noises such that the noisy $\mathrm{fBm}$ had an SNR value of $5 \mathrm{~dB}$. We can see that all denoised results are visually acceptable. In the following, we demonstrate two applications for fbm image parameter estimation and denoising. In both applications, we used the Haar wavelet to process the data. 


\subsection{Application 1 : Coastline detection}

The first application of $\mathrm{fBm}$ image denoising is a model of a terrain surface. In order to identify the coastline, we set those pixel values below a certain threshold to black as if they were below sea level. For example, Fig. $7(\mathrm{a})$ is an $\mathrm{fBm}$ image with $\alpha=0.5$, and Fig. 7(b) is the result of coastline detection. If the image is added with white noise, then simple thresholding cannot identify the coastline well. This is clearly shown in Fig. 7(c), where 5dB noise was added to the image shown in Fig. 7(a). One can observe many dotted noises, and that the coastline cannot be identified clearly. In Fig. 7(d), we show the result of coastline detection on the denoised image using our algorithm. It is a smoothed version of the original coastline shown in Fig. 7(b), but shows essential topographical features comparing to $7(\mathrm{c})$. For this application, one may simply filter the noisy fBm image with a low-pass filter and threshold the resultant image for the denoised coastline. However, the parameters of the low-pass filter are usually hard to determine and the resultant smoothed image is not an $\frac{1}{f}$ process. Besides, the knowledge of the $\mathrm{fBm}$ and noise is not used in this approach. Therefore, low-pass filtering is not a suitable solution compared with our denoising approach.

\subsection{Application 2 : Texture segmentation}

The estimated fractal parameter $\alpha$ can be used as a useful feature for texture segmentation and classification. In this subsection we will demonstrate its application in texture segmentation. Fig. 8 (a) shows a $450 \times 380$ image of natural scenes, which by human eyes can be classified into three clusters : a sky, a cloud, and a mountain surface. We used a small sliding window of size $32 \times 32$ to estimate the scaling exponent $\alpha$, and the center pixel of this window was assigned this estimated $\alpha$ value as its local feature. This fractal feature was computed for each pixel, then this feature image was clustered to obtain the segmented image. A Gaussian filter of variance 6 was used to smooth the resultant feature images. Then, we applied c-mean algorithm to classify each pixel 
to one cluster, assuming that the number of clusters was given as a priori knowledge. The classified pixels were given gray level $\mathrm{N}$ which was equal to their cluster number. This clustered image is shown in Fig. 8(b). Although the cloud can be modeled as an $\mathrm{fBm}$, there are still regions of smooth gray values inside the cloud and these regions are mis-classified. The mountain surface and the sky form another areas with different degrees of coarseness. This leads to different fractal parameter $\alpha$ in these areas.

Fig. $9(\mathrm{a})$ shows a $512 \times 512$ texture mosaic created by three $\mathrm{fBm}$ images with different scaling exponents $\alpha$ : in the upper $256 \times 512$ is an fBm image with $\alpha=0.8$, in the lower left corner is a $256 \times 256 \mathrm{fBm}$ image with $\alpha=0.5$, and in the lower right corner is a $256 \times 256 \mathrm{fBm}$ image with $\alpha=0.2$. One can easily see the texture boundaries. They are not detectable by an edge detection method; too many edge points will be found due to the singular behavior of an $\mathrm{fBm}$. According to our previous experimental result in Fig. 1, in the case of clean $\mathrm{fBm}$ parameter estimation, the degree of the RMS error is below $10^{-1}$ for window size above or equal to $32 \times 32$. Therefore, We used sliding window of size $32 \times 32$ to estimate the fractal parameter of the clean $\mathrm{fBm}$ mosaic. It had been reported that the fractal feature alone cannot segment texture well [9], especially in the case of noisy environment, in which the parameters cannot be precisely estimated with only local data. So we added the fracal power parameter $\sigma_{\alpha}^{2}$ in (3) as another feature. $\sigma_{\alpha}^{2}$ could be converted from $K_{p}$ in our parameter estimation process, or derived as follows. From the formula of fBm [9],

$$
\operatorname{var}\left[f_{\alpha}(u+x, v+y)-f_{\alpha}(u, v)\right]=\sigma_{\alpha}^{2}\left(x^{2}+y^{2}\right)^{\alpha},
$$

first we compute the $\mathrm{x}$ - and $\mathrm{y}$ - directed increments of the $\mathrm{fBm}$ image, that is, $I_{x}(u, v)=$ $f_{\alpha}(u, v)-f_{\alpha}(u-1, v)$ and $I_{y}(u, v)=f_{\alpha}(u, v)-f_{\alpha}(u, v-1)$. Then we calculate the average energy of each of the two increments over a window as the approximation to $\sigma_{\alpha}^{2}$. These two quantities can be used as local features for the middle pixel in the window. A Gaussian filter of variance 4 was used for smoothing. Then, c-mean algorithm was used to classify the feature images. This clustered image is shown in Fig. 9(c). The 
major segmentation errors happened in the texture boundaries, in which the parameter estimation is inaccurate.

White noise was added to the fBm mosaic in Fig. 9(a) such that the SNR is 10dB. This noisy fBm mosaic is shown in Fig. 9(b). From previous experiments in Fig. 2, window size must be greater than $64 \times 64$ to achieve better parameter estimation. We thus chose sliding window of size $64 \times 64$. The scaling exponent and the fractal power parameter for each pixel were also estimated. Similar Gaussian smoothing of variance 6 and c-mean clustering method were applied in the noisy fBm mosaic. The clustered result is shown in Fig. 9(d). A more severe segmentation error occurs in the texture boundaries. Based on this segmentation result, we will estimate the $\mathrm{fBm}$ mosaic. We identified the texture boundaries of the noisy fBm mosaic and partitioned them into three rectangular sub-images. Then, we applied our parameter estimation method to each sub-image for the parameters $\alpha, K_{p}$, and $K_{n}$. We obtained $\sigma_{\alpha}{ }^{2}$ and $\sigma_{n}{ }^{2}$ from the estimated $K_{p}$ and $K_{n}$ at each sub-image by using the Equations (6) and (8), respectively. Finally, the denoised sub-images were obtained by using our proposed Wiener filtering method. The denoised fBm mosaic is shown in Fig. 9(e). The SNR of the denoised $\mathrm{fBm}$ mosaic is about $17.21 \mathrm{~dB}$. Thus, we have about $7 \mathrm{~dB}$ gain from the segmentation and denoising process.

\section{Conclusion}

We have showed that the wavelet transform of a $2-\mathrm{D} \mathrm{fBm}$ at each scale is WSS. A new fractal estimation method, based on the decay of the variance of the wavelet transform of a noisy fBm image across scales, has been proposed. This new method allows estimation of the fractal parameter on small image blocks, and outperforms many conventional fractal parameter algorithms on small images, where the fractal parameter is obtained by averaging the 1-D results in many directions using 1-D fractal estimation algorithm. 
For the estimation of a denoised image, a Wiener filter was applied to the noisy wavelet transform on each scale. Then, a smoothed "denoised" image was obtained after applying the inverse wavelet transform. We have shown that the averaged power

spectrum of the estimated image is isotropic and is a near $\frac{1}{f}$ process. Finally, we demonstrated our algorithms on the applications of coastline detection and texture segmentation. Further extension of this work to wider classes of scaling processes is under investigation.

\section{References}

[1] Dimitri P. Bertsekas, Constrained optimization and Lagrange multiplier methods. New York, Academic Press 1982.

[2] C. Chen, J. S. Deponte and M. D. Fox, "Fractal feature analysis in medical imaging," IEEE Transaction on Medical Imaging, MI-8, pp. 133-142, 1989.

[3] N. Delprat, B. Escudie, P. Guillemain, R. Kronland-Martinet, P. Tchamitchian, and B. Torresani, "Asymptotic wavelet and Gabor analysis: extraction of instantaneous frequencies," IEEE Transaction on Information Theory, Vol. 38, No. 2, 1992.

[4] P. Flandrin, "On the spectrum of fractional Brownian motions," IEEE Trans. Inform. Theory, Vol. IT-35, pp. 197-199, Jan. 1989.

[5] P. Flandrin, "Wavelet analysis and synthesis of fractional Brownian motion," IEEE Transaction on Information Theory, Vol. 38, No. 2, March 1992.

[6] W. L. Hwang and S. Mallat, "Characterization of self-similar multifractals with wavelet maxima," Applied and Computational Harmonic Analysis, 1, pp. 316-328, 1994. 
[7] W. L. Hwang, "Estimation of fractional Brownian motion embedded in a noisy environment using non-orthogonal wavelets," IEEE Trans. Signal Processing, Vol. 47, No. 8, Aug. 1999.

[8] L. Kaplan and C.-C. Kuo, "Fractal estimation from noisy measurements via discrete fractional Gaussian noise and the Haar basis," IEEE Transactions on Signal Processing, Vol. 41, No. 12, December 1993.

[9] L. Kaplan, and C.-C. Kuo, “Texture segmentation via Haar fractal feature estimation," J. Visual Comm. and Image Representation, Vol. 6, No. 4, Dec. 1995.

[10] L. Kaplan, and C.-C. Kuo, "Texture roughness analysis and synthesis via extended self-similar (ESS) model," IEEE Transactions on Image Processing, Vol. 17, No. 11, Nov. 1995.

[11] L. Kaplan and C.-C. Kuo, "An improved method for 2-D self-similar image synthesis," IEEE Transactions on Image Processing, Vol. 5, No. 5, May 1996.

[12] T. Lundhal, W. J. Ohley, S. M. Kay, and R. Siffert, "Fractional Brownial motion: an ML estimator and its application to image texture," IEEE Transaction on Medical Imaging, Vol. MI-5, No. 3, September 1986.

[13] S. Mallat and W. L. Hwang, "Singularity detection and processing with wavelets," IEEE Transaction on Information Theory, Vol. 38, No. 2, March 1992.

[14] S. Mallat and S. Zhong, "Characterization of signals from multi-scale edges," IEEE Transaction on Pattern Analysis and Machine Intelligence, Vol. 14, No. 7, 1992.

[15] B. Mandelbrot and H. Van Ness, "Fractional Brownian motions, fractional noises and applicatioins," SIAM Review, Vol. 10, Oct. 1968.

[16] A. P. Pentland, "Fractal-based description of natural scenes," IEEE Trans. Pattern Anal. Machine Intell., Vol. PAMI-6, pp. 661-674, Nov. 1984. 
[17] K. Perlin, "Hypertexture," Computer Graphics, Vol. 23, No. 23, July 1989.

[18] Peter J. Rousseeuw, "Least median of squares regression," J. of Amer. Stat. Assoc., Vol 79, No. 388, Dec. 1984.

[19] Z. Cvetkovic and M. Vetterli, "Discrete-Time wavelet extrema representation: design and consistent reconstruction," IEEE Trans. Signal Processing, Vol. 43, No. 3, March 1995.

[20] G. W. Wornell, "A Karhunen-Loeve-like expansion for 1/f processes via wavelet," IEEE Trans. Inform. Theory, Vol. IT-36, pp. 859-861, July 1990.

[21] G. W. Wornell and A. Oppenheim, "Estimation of fractal signals from noisy measurements using wavelet," IEEE Transaction on Signal Processing, March 1992.

[22] Stephan Hoefer, Frank Heil, Madhukar Pandit, Ramdas Kumaresan, "Segmentation of textures with different roughness using the model of isotropic twodimensional fractional Brownian motion," ICASSP, 1993.

[23] I.S. Reed, P.C. Lee and T.K. Truong, "Spectral representation of fractional Brownian motion in n-dimensions and its properties," IEEE Trans. on Inform. Theory, Vol. 41, No. 5, pp. 1439, 1995.

[24] B.F. Wu and Y.L. Su, "On stationarizability for nonstationary 2-D random fields using discrete wavelet transforms," IEEE Trans. on Image Processing, pp. 13591366, Sep., 1998.

[25] A. Arneodo, N. Decoster, and S.G. Roux, "Intermittency, log-normal statistics, and multifractal cascade process in high-resolution satellite images of cloud structure," Physical Review Letters, Vol. 83, No. 6, pp. 1255-1258, Aug. 1999. 

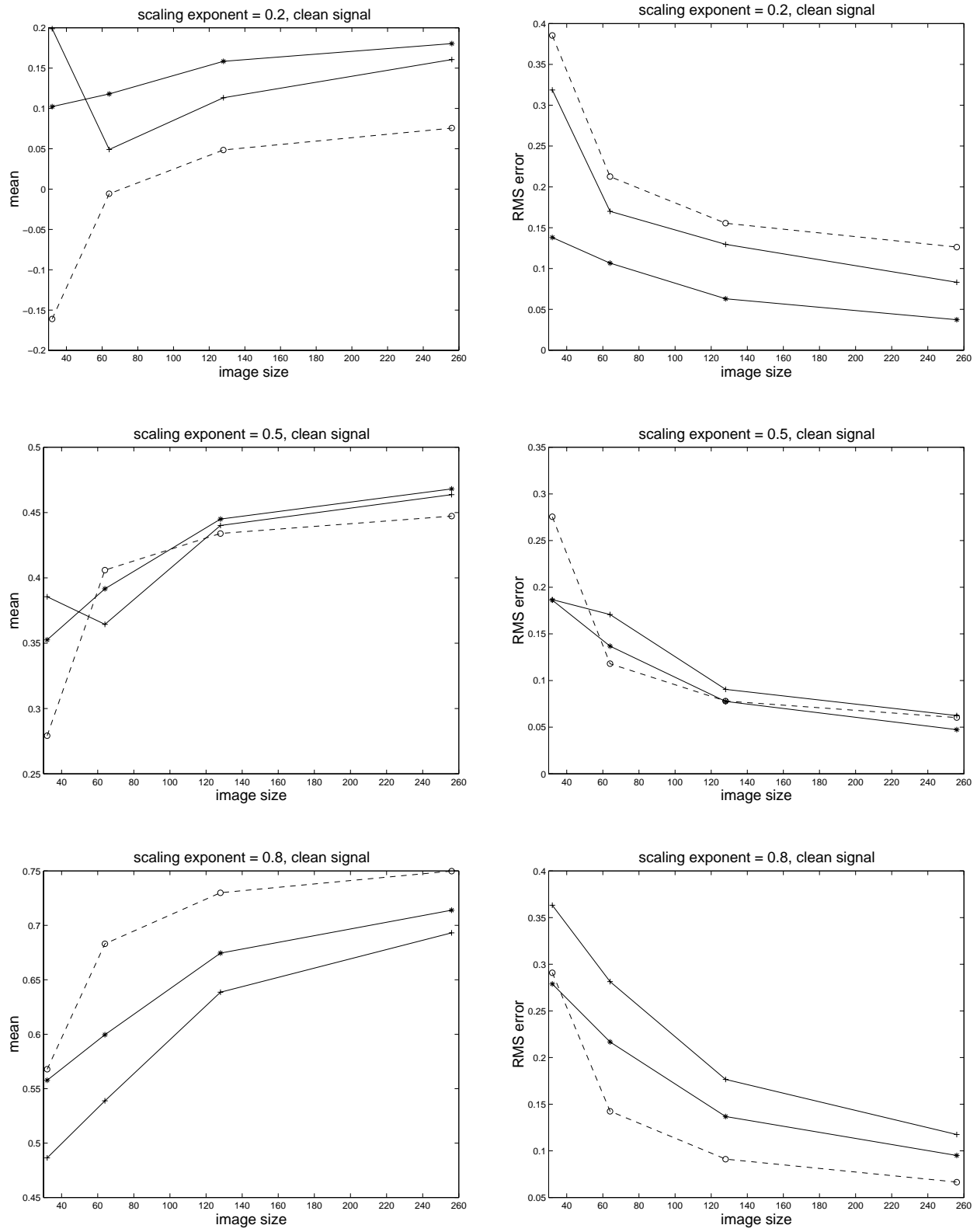

Figure 1: The mean and RMS error of the scaling exponent estimation from 64 realizations of clean fBm images with various sizes. "*", and "+" indicate the results obtained by using the proposed 2-D estimation method with Haar and Mallat wavelet, respectively. "o" denotes the result obtained by using 1-D WO's method with Haar wavelet. Top: Estimation of $\alpha=0.2$. Middle: Estimation of $\alpha=0.5$. Bottom: Estimation of $\alpha=0.8$. 

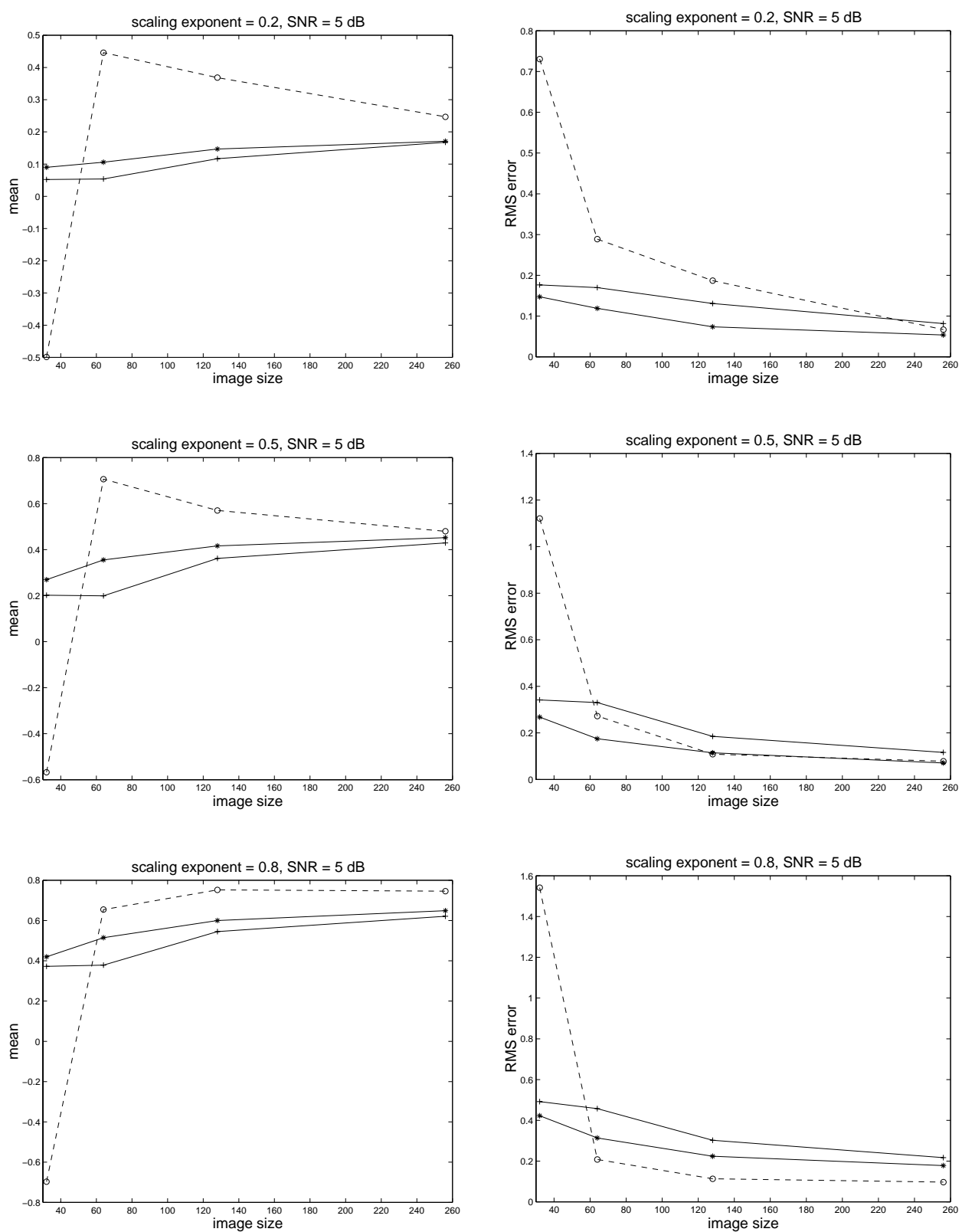

Figure 2: The mean and RMS error of the scaling exponent estimation from 64 realizations of noisy $\mathrm{fBm}$ images with various sizes. Noise was added to image such that $\mathrm{SNR}=5 \mathrm{~dB}$. "*", and "+" indicate the results obtained by using the proposed 2-D method with Haar and Mallat wavelet, respectively. "o" denotes the result obtained by using 1-D WO's method with Haar wavelet. Top: Estimation of $\alpha=0.2$. Middle: Estimation of $\alpha=0.5$. Bottom: Estimation of $\alpha=0.8$. 


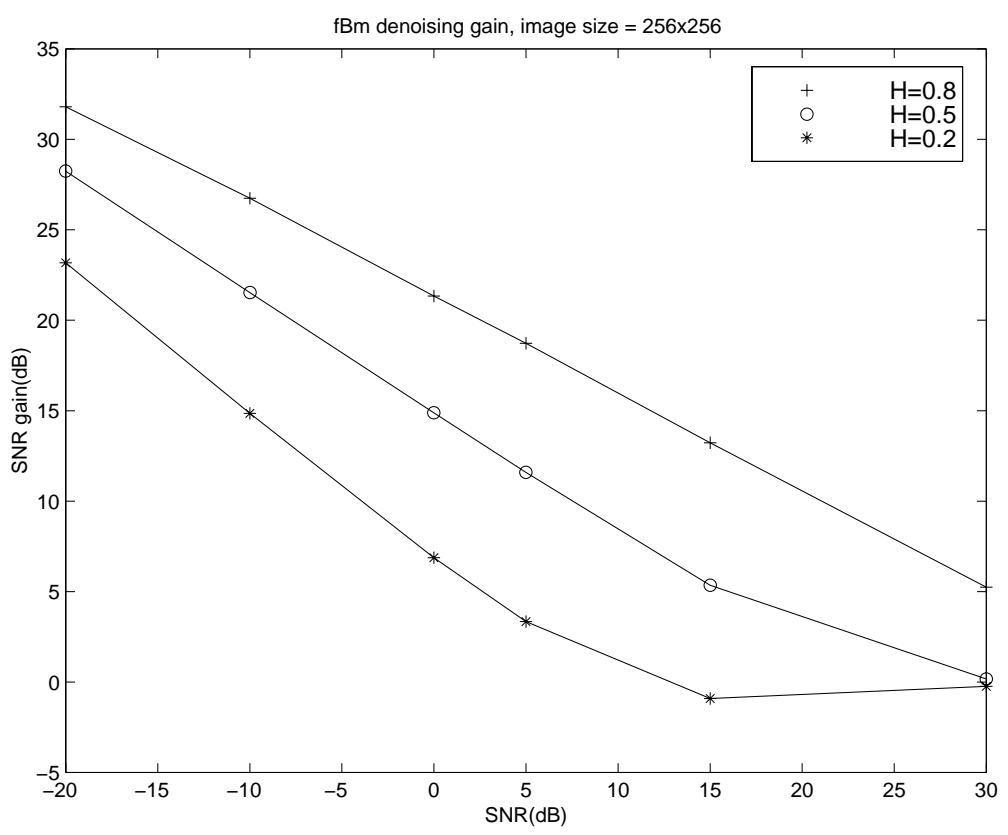

(a)

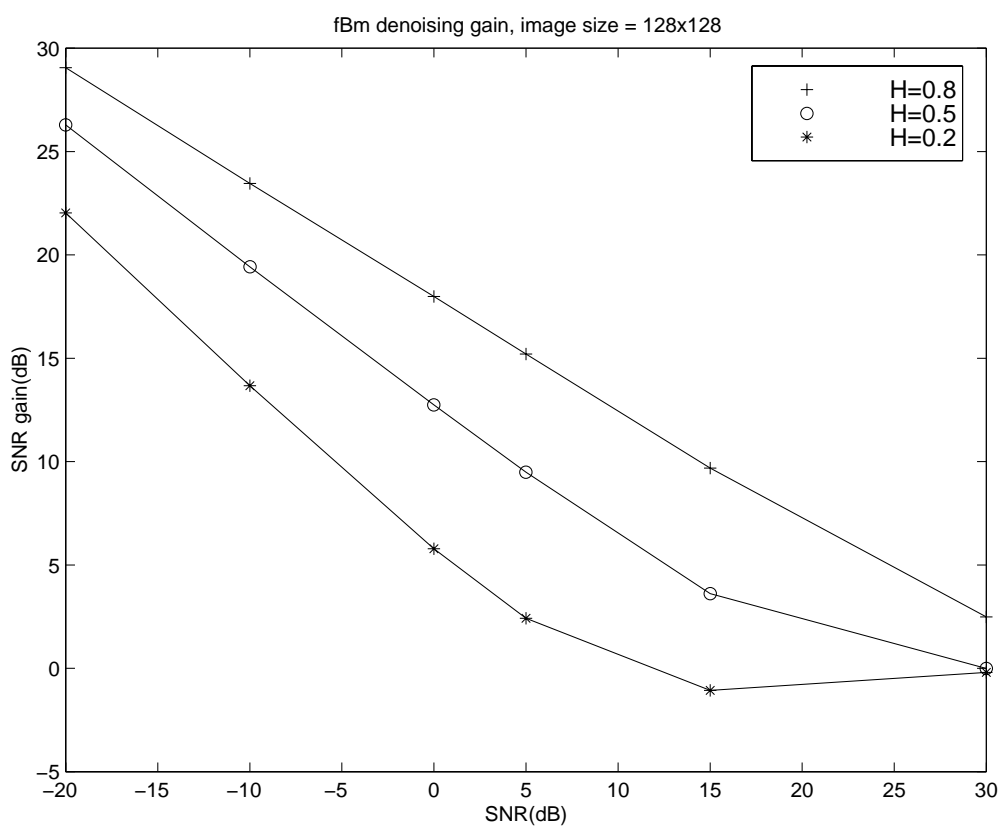

(b)

Figure 3: The SNR gain from denoising the image with various SNR using Mallat wavelet. (a): image of size $256 \times 256$. (b): image of size $128 \times 128$. "+", "o", and "*" indicate the results obtained of fBm with $\alpha=0.8,0.5$. and 0.2 , respectively. 

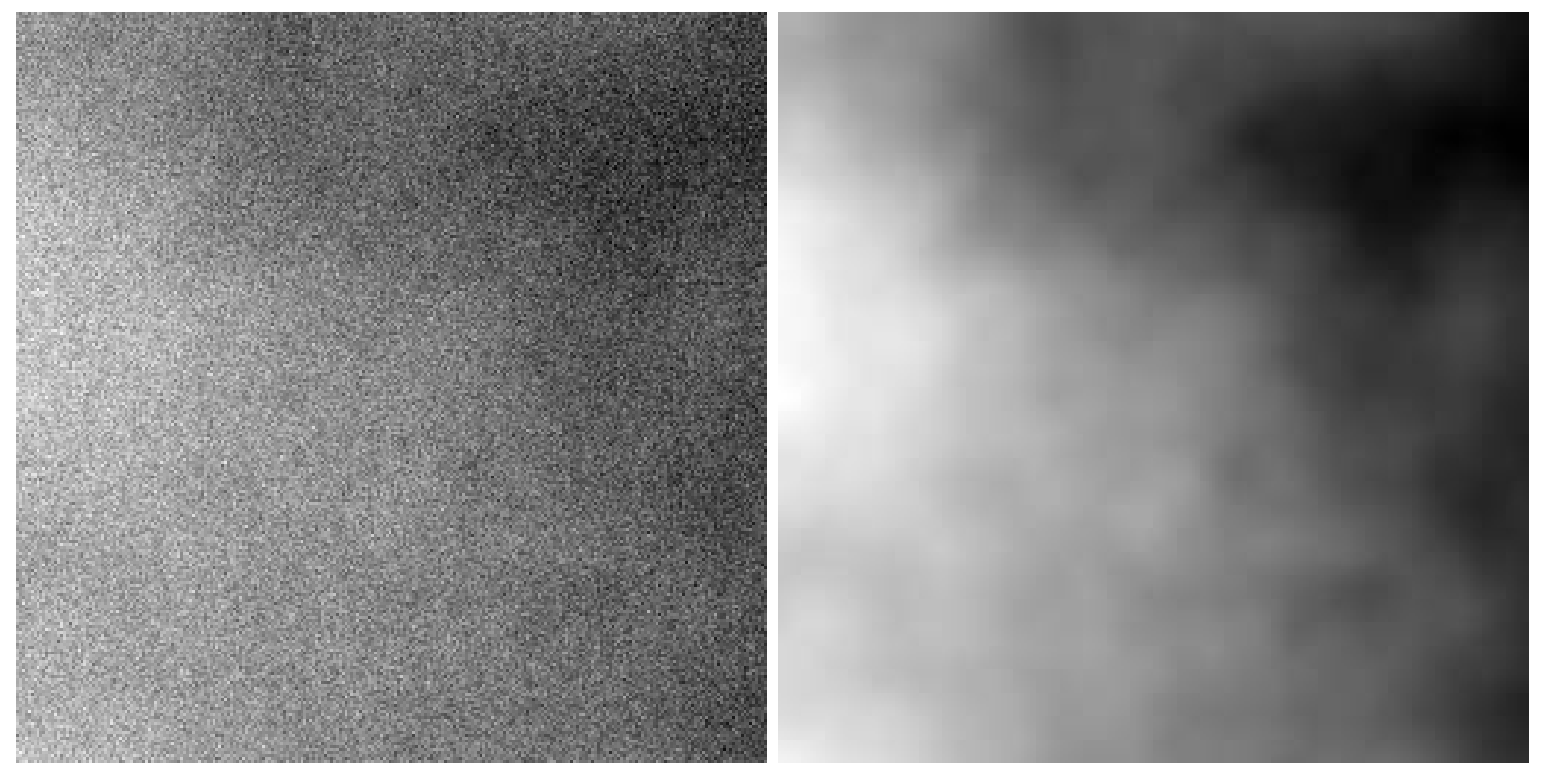

Figure 4: Image denoising example. Top figure : $256 \times 256 \mathrm{fBm}$ image with $\alpha=0.8$. Bottom left : noisy fBm with $\mathrm{SNR}=5 \mathrm{~dB}$. Bottom right : denoised $\mathrm{fBm}$ image with SNR gain 21.12dB. 


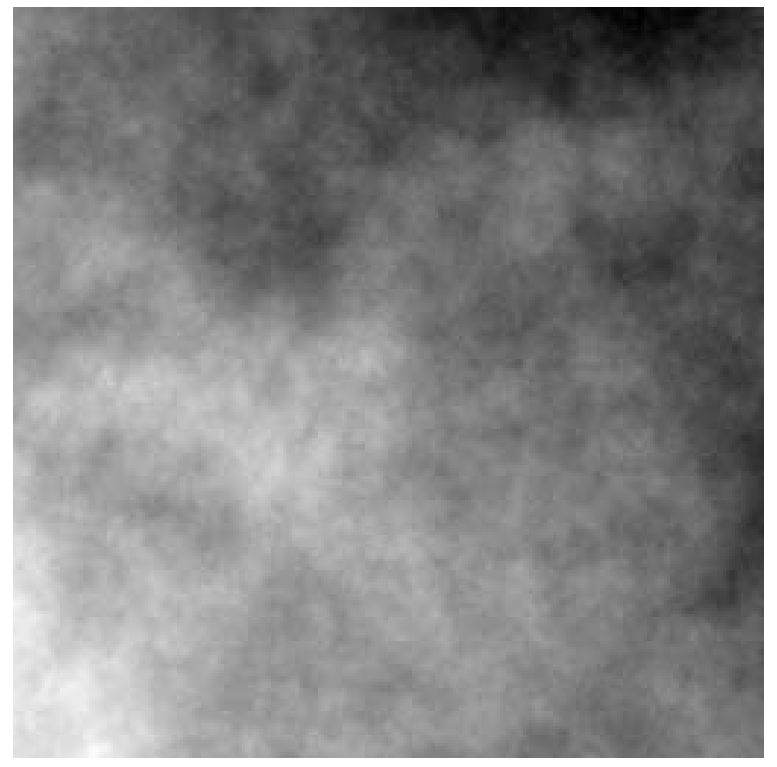

(a)
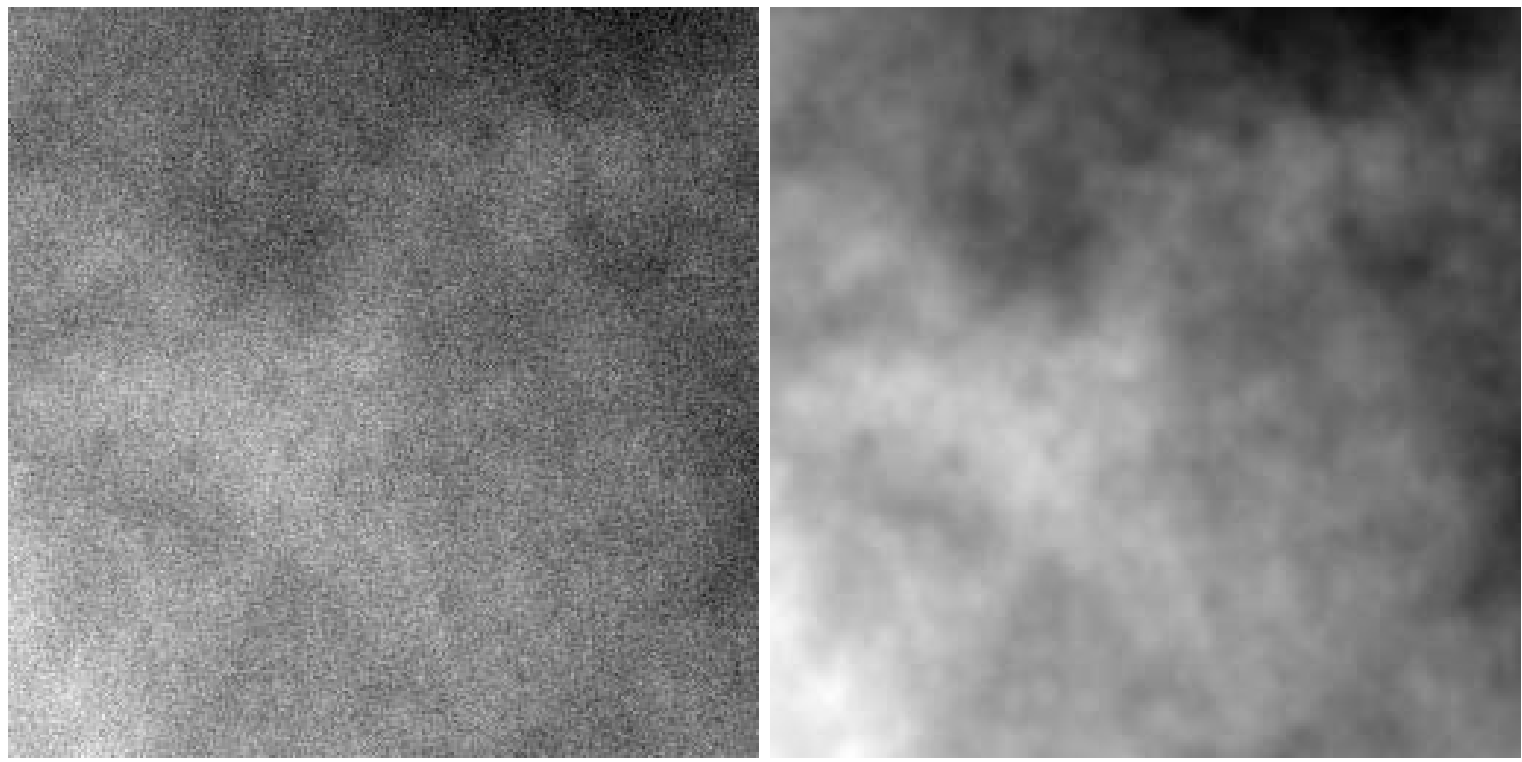

Figure 5: Image denoising example. Top figure : $256 \times 256 \mathrm{fBm}$ image with $\alpha=0.5$. Bottom left : noisy fBm with $\mathrm{SNR}=5 \mathrm{~dB}$. Bottom right $:$ denoised fBm image with SNR gain 13.33dB. 

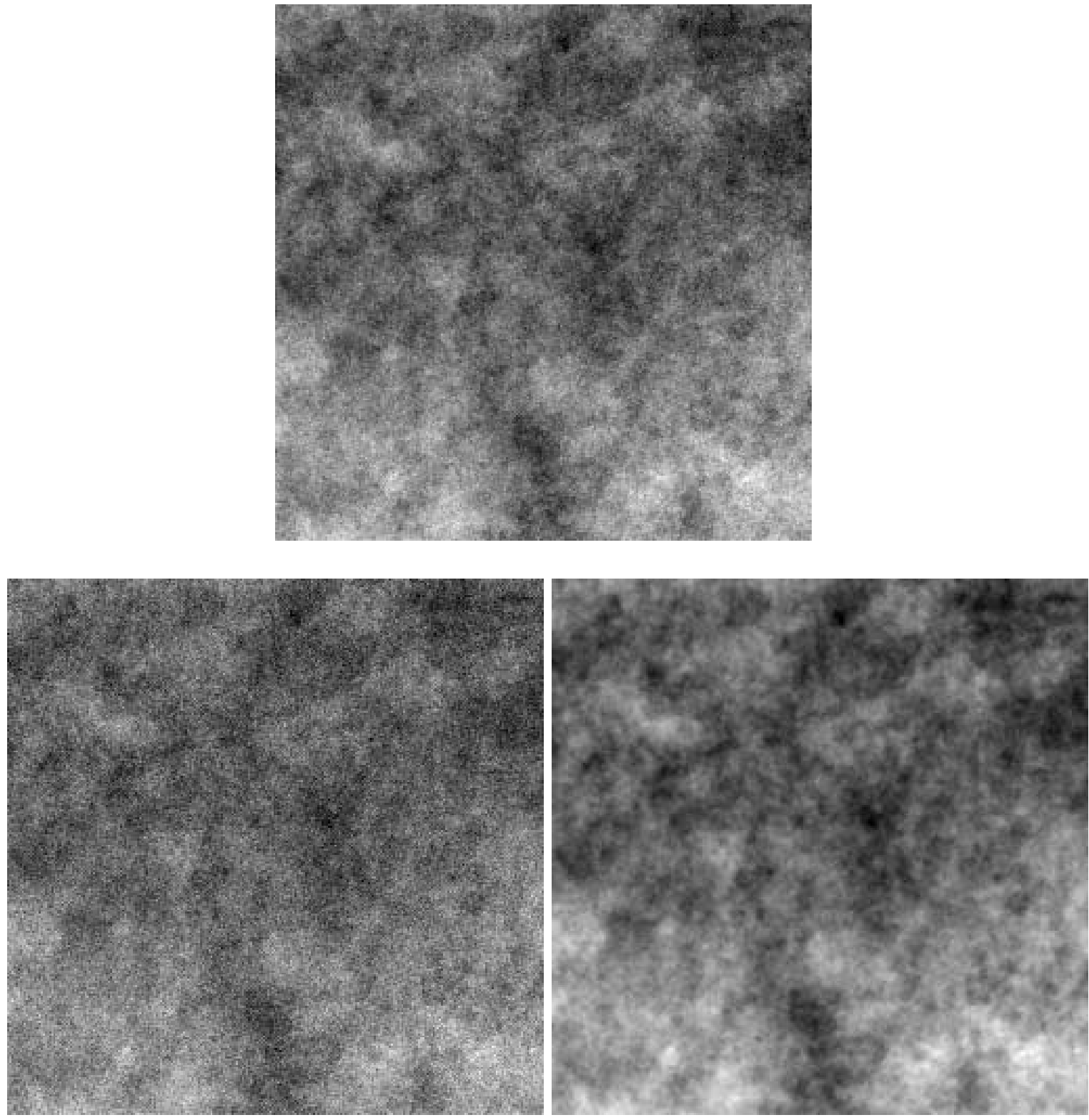

Figure 6: Image denoising example. Top figure : $256 \times 256 \mathrm{fBm}$ image with $\alpha=0.2$. Bottom left : noisy fBm with $\mathrm{SNR}=5 \mathrm{~dB}$. Bottom right : denoised $\mathrm{fBm}$ image with SNR gain $3.48 \mathrm{~dB}$. 


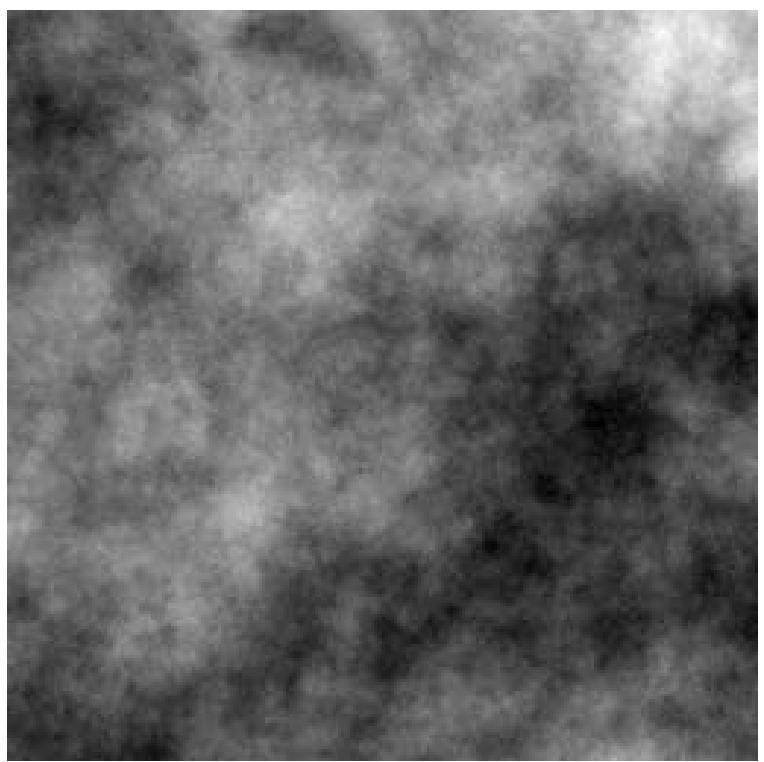

(a)

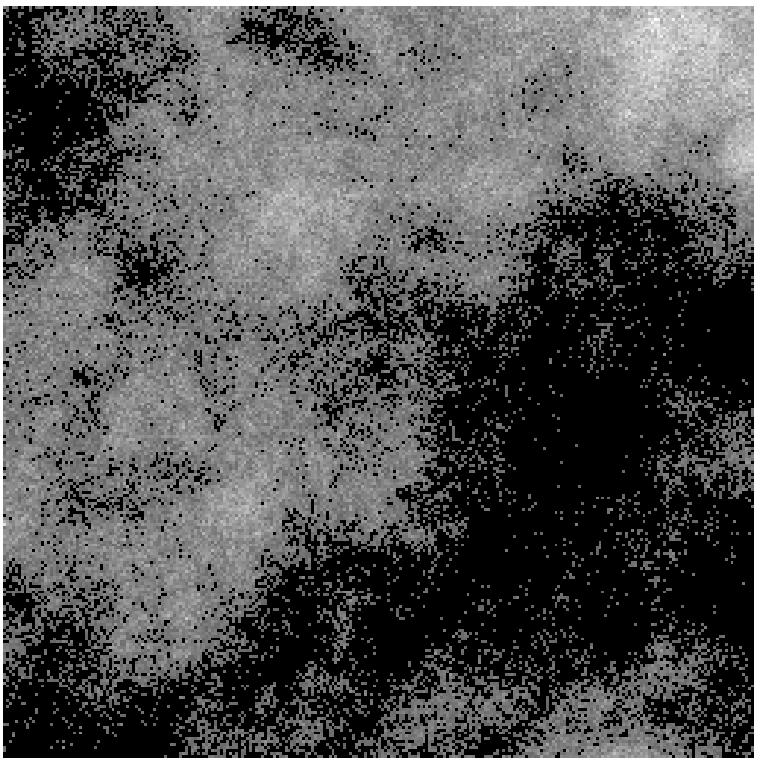

(c)

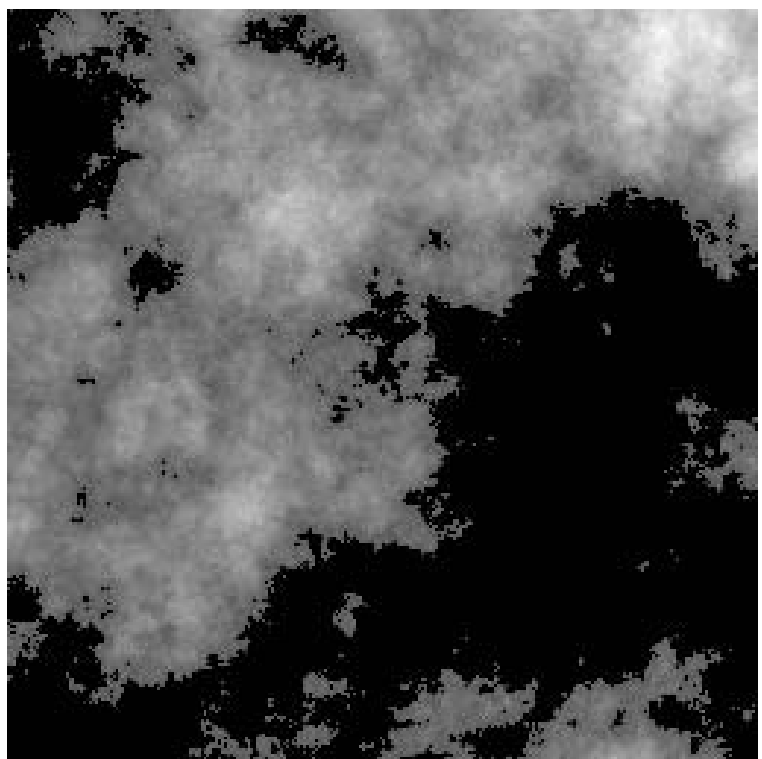

(b)

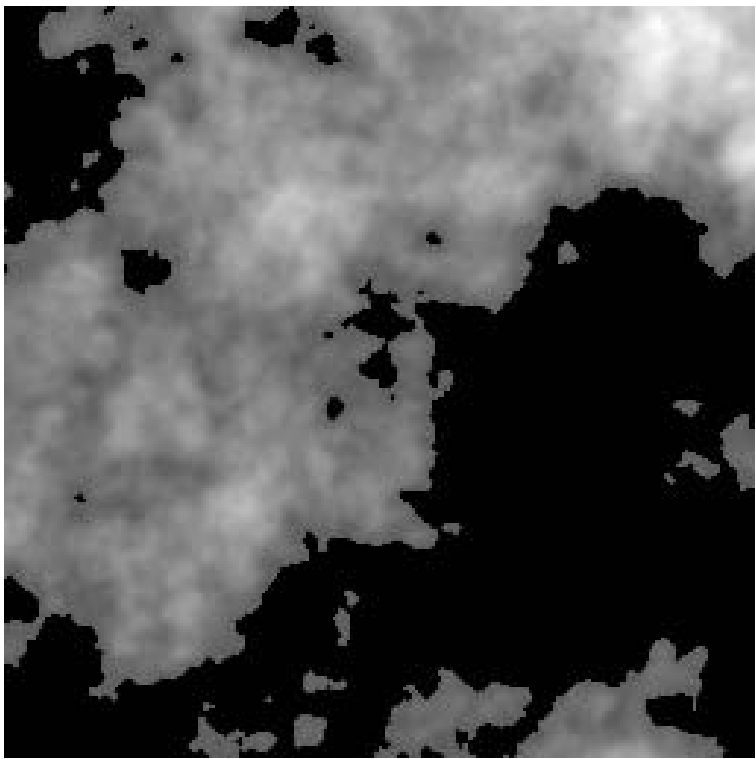

$(\mathrm{d})$

Figure 7: Example of coastline detection. (a): original 256 × $256 \mathrm{fBm}$ image with $\alpha=0.5$. (b): coastline detection of original fBm image. (c): coastline detection of the noisy $\mathrm{fBm}$ with $\mathrm{SNR}=5 \mathrm{~dB}$. (d): coastline detection of the denoised $\mathrm{fBm}$ image. 


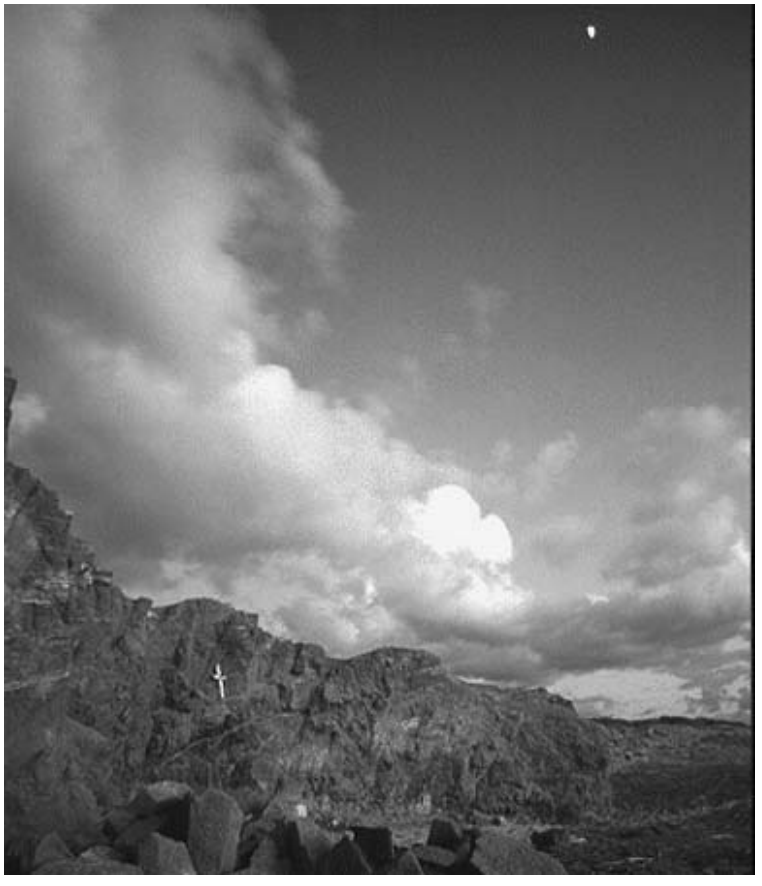

(a)

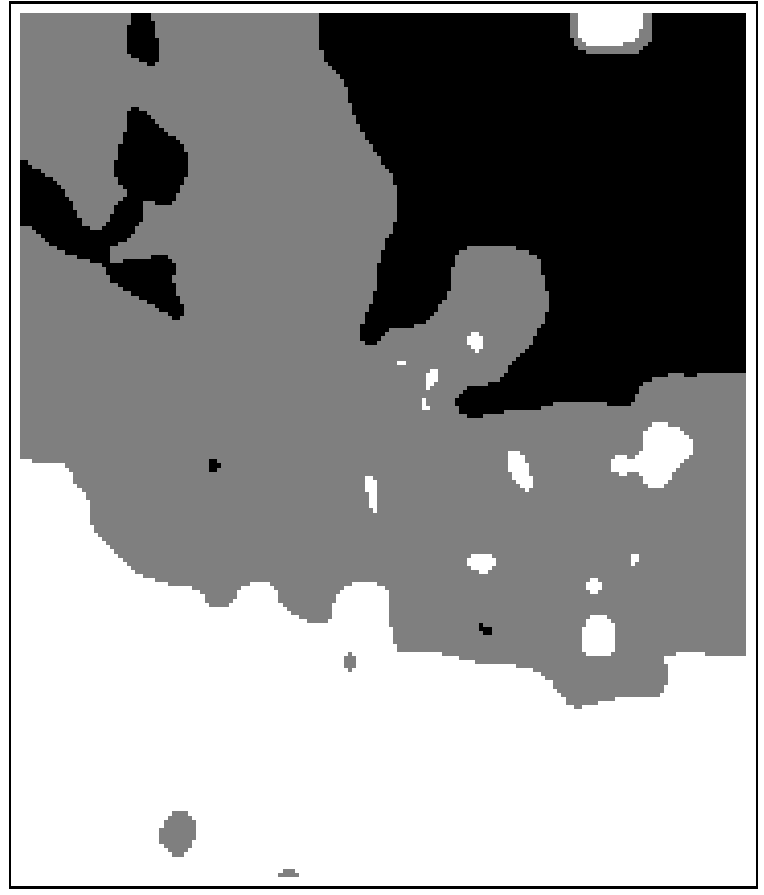

(b)

Figure 8: Segmentation of natural scenes. (a): Original $450 \times 387$ photograph () Aris Entertainment, 1991). (b): Texture segmentation result. 


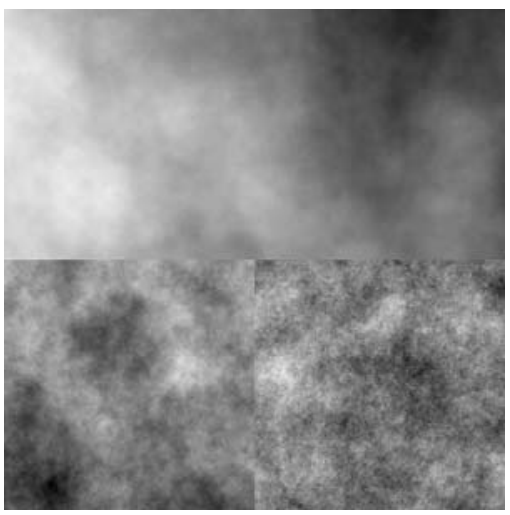

(a)

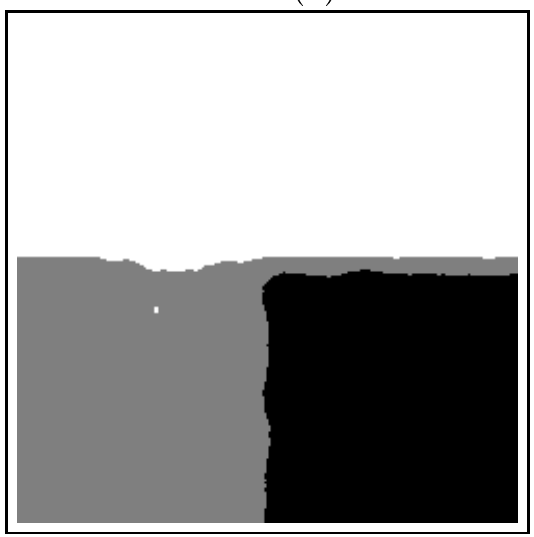

(c)

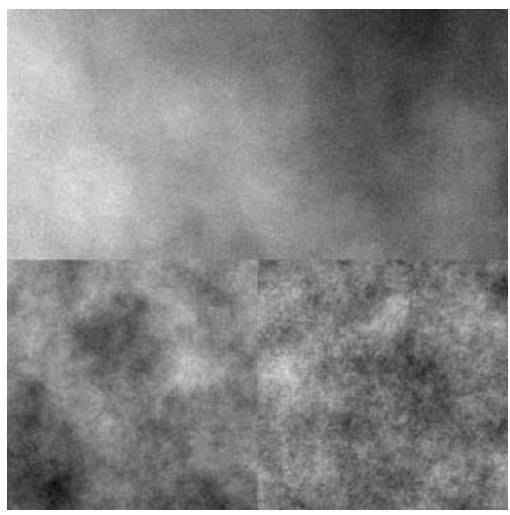

(b)

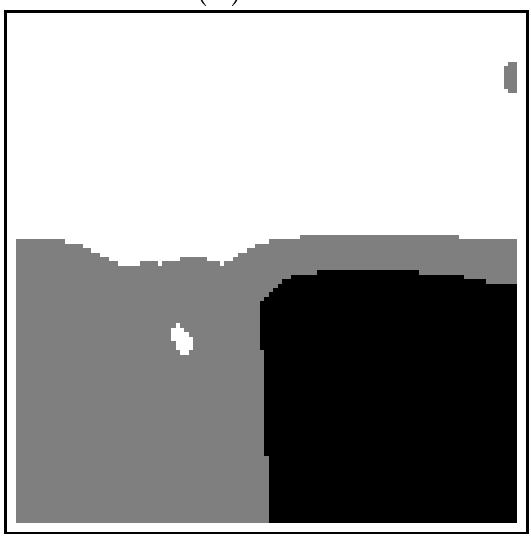

(d)

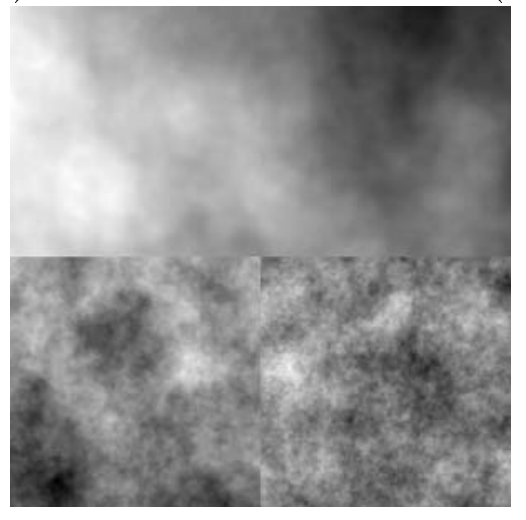

(e)

Figure 9: Application of texture segmentation and denoising. (a): Original $512 \times 512$ fBm image mosaic. (b): Noise was added to (a) such that SNR $=10 \mathrm{~dB}$. (c): Texture segmentation result of (a). (d): Texture segmentation result of (b). (e): Denoised image of (b) according to the segmentation result of (d). 